\title{
Crescimento do Algodoeiro Submetido ao Herbicida TRIFLOXYSULFURON-SODIUM ${ }^{1}$
}

\author{
Growth of Cotton Treated with Trifloxysulfuron-Sodium
}

FREITAS, R.S. ${ }^{2}$, TOMAZ, M.A. ${ }^{3}$, FERREIRA, L.R. ${ }^{4}$, BERGER, P.G. ${ }^{4}$, PEREIRA, C.J. ${ }^{5}$ e CECON, P.R. ${ }^{6}$

\begin{abstract}
RESUMO - Este trabalho foi realizado com o objetivo de avaliar o efeito do trifloxysulfuron-sodium no crescimento do algodoeiro cultivar Fabrika. As plantas foram submetidas ou não a 7,5 $\mathrm{g} \mathrm{ha}^{-1}$ de trifloxysulfuron-sodium, aos 19 dias após a emergência, quando estavam com quatro folhas verdadeiras (estádio $\mathrm{V}_{4}$ ). Os índices de crescimento das plantas foram determinados aos $0,7,14$, 24, 36, 52 e 73 dias após aplicação do herbicida (DAA). O delineamento experimental foi o inteiramente casualizado, com quatro repetições. A intoxicação causada pelo herbicida foi maior aos 20 dias após sua aplicação (DAA), com 35\% de dano. O acúmulo de matéria seca do caule principal, do total de folhas, de folhas do ramo principal, da parte aérea e a área foliar aumentaram com o desenvolvimento da cultura. Entretanto, o ponto de máximo crescimento para essas características foi atingido mais precocemente nas plantas-controle, exceto para área foliar total. De modo geral, a taxa de crescimento absoluto, a taxa de crescimento relativo e a taxa assimilatória líquida foram menores nas avaliações iniciais, nas plantas submetidas ao herbicida. As plantascontrole apresentaram redução mais acentuada na razão de área foliar que as submetidas ao herbicida. O trifloxysulfuron-sodium também causou atraso no crescimento das maçãs, que apresentaram metade do acúmulo de matéria seca em relação às plantas-controle, aos 52 DAA; todavia, a produção de algodão em caroço foi semelhante entre os tratamentos. O trifloxysulfuronsodium influenciou negativamente o crescimento do algodoeiro, principalmente nas primeiras semanas após sua aplicação. Entretanto, a rápida recuperação da planta tratada possibilitou produção de algodão em caroço semelhante àquela obtida com as plantas-controle.
\end{abstract}

Palavras-chave: Gossypium hirsutum, taxa de crescimento absoluto, taxa assimilatória líquida, taxa de crescimento relativo.

\begin{abstract}
This work was carried out under greenhouse conditions to evaluate the effect of trifloxysulfuron-sodium on the growth of the cotton cultivar Fabrika. The plants were treated or not treated with $7.5 \mathrm{~g}$ a.i. ha $\mathrm{a}^{-1}$ of trifloxysulfuron-sodium 19 days after emergence, when they had four true leaves (stage V4). Plant growth indices were determined at $0,7,14,24,36,52$, and 73 days after application - DAA) of the herbicide. The experiment was arranged in a completely randomized block design, with four replications. Herbicide-induced plant poisoning was strongest at 20 DAA with 35\% damage. Dry matter accumulation of the main stem, total leaves, main branch leaves, and shoot and leaf area increased with cotton development. However, the maximum growth point for these characteristics was reached earlier by the control-plants, except for total foliar area. In general, the absolute and relative growth rates and net assimilation rate were lowest at the initial evaluations, in the herbicide-treated plants. The control-plants had more noticeable reduction in the leaf area rate than the herbicide-treated plants. Trifloxysulfuron-sodium also caused a delay in the growth of bolls, which presented half of the dry matter accumulation 52 DAA, compared to the control plants; however, cotton productivity was similar among the treatments. Trifloxysulfuronsodium influenced cotton development negatively, mainly during the first weeks after application but it did not affect cotton productivity.
\end{abstract}

Keywords: Gossypium hirsutum, absolute growth rate, net assimilation rate, relative growth rate, weed.

1 Recebido para publicação em 15.3.2005 e na forma revisada em 24.2.2006.

2 Pesquisador Científico I/APTA - Pólo Regional do Noroeste Paulista - Florestal, Km 4, Caixa Postal 61, 1550-000 Votuporanga-SP, <freitas@aptaregional.sp.gov.br>; ${ }^{3}$ D.S. pelo Dep. de Fitotecnia da Universidade Federal de Viçosa - DFT/UFV, 36570.000 Viçosa-MG; ${ }^{4}$ Professor do Dep. de Fitotecnia - DFT/UFV; ${ }^{5}$ Estudante de mestrado da UFV; ${ }^{6}$ Prof. do Dep. de Informática - DPI/UFV. 


\section{INTRODUÇÃO}

O trifloxysulfuron-sodium é um herbicida do grupo químico das sulfoniluréias, recomendado para o manejo de plantas daninhas na cultura do algodoeiro, em pós-emergência total, por ser eficiente no controle de importantes espécies de plantas daninhas dicotiledôneas e seletivo à cultura. Entretanto, tem-se observado que esse herbicida causa toxidez ao algodoeiro (Barber et al., 2002; Schraer et al., 2002; Freitas et al., 2004), podendo, inclusive, ser superior a $60 \%$ (Burke et al., 2002; Porterfield et al., 2002a). Toxidez superior a $60 \%$ em plantas de algodão foi verificada por Porterfield et al. (2002a) em dois dos cinco locais estudados, quando o trifloxysulfuronsodium $\left(7,5 \mathrm{~g} \mathrm{ha}^{-1}\right)$ foi aplicado estando a planta no estádio fenológico de três a quatro folhas e com 10 a $15 \mathrm{~cm}$ de altura. A maior intoxicação das plantas pelo herbicida ocorreu quando estas estavam menos desenvolvidas.

Neste trabalho foi utilizada a análise de crescimento para quantificar os efeitos do herbicida trifloxysulfuron-sodium no algodoeiro. Ela gera informações relevantes nesse tipo de estudo, uma vez que descreve as mudanças na produção vegetal ao longo do tempo. A análise de crescimento de comunidades vegetais é um dos primeiros passos na análise de produção primária, caracterizando-se como o elo entre o simples registro do rendimento das culturas e a análise destas por meio de métodos fisiológicos. Pode, ainda, ser utilizada para conhecer a adaptação ecológica das plantas a novos ambientes, a competição interespecífica, os efeitos de sistemas de manejo e a capacidade produtiva de diferentes genótipos (Kvet et al., 1971). Ademais, a análise de crescimento representa a referência inicial na análise de produção das espécies vegetais e seu uso requer informações que podem ser obtidas sem a necessidade de equipamentos sofisticados (Pereira \& Machado, 1987).

Desse modo, objetivou-se com este trabalho avaliar o crescimento do algodoeiro submetido ao trifloxysulfuron-sodium em pós-emergência.

\section{MATERIAL E MÉTODOS}

O experimento foi realizado em casa de vegetação, no período de 23 de janeiro a 10 de junho de 2004. As plantas foram cultivadas em vasos contendo $12 \mathrm{~kg}$ de Argissolo Vermelho-Amarelo câmbico (Tabela 1), fase terraço, previamente peneirado e adubado com $\mathrm{P}_{2} \mathrm{O}_{5}$ e $\mathrm{K}_{2} \mathrm{O}$ a $1,260 \mathrm{~kg} \mathrm{~m}^{-3}$ e $0,230 \mathrm{~kg} \mathrm{~m}^{-3}$, respectivamente. Foram feitas, também, adubações de cobertura, com macro e micronutrientes, segundo recomendações técnicas para a cultura (Silva, 1999), considerando o maior nivel de produtividade. Foram semeadas 10 sementes do cultivar Fabrika em cada vaso, com o objetivo de obter uma planta uniforme por vaso, após o desbaste.

As plantas foram submetidas ou não a $7,5 \mathrm{~g} \mathrm{ha}^{-1}$ de trifloxysulfuron-sodium aos 19 dias após a emergência, quando estavam com quatro folhas verdadeiras, ou seja, estádio $\mathrm{V}_{4}$ (Murur \& Ruano, 2002). As plantas foram coletadas aos $0,7,14,24,36,52$ e 73 dias após a aplicação, sendo seus índices de crescimento avaliados nesses períodos, para ambos os tratamentos. O delineamento experimental foi inteiramente casualizado, com quatro repetições.

O herbicida foi aplicado com pulverizador costal pressurizado a $\mathrm{CO}_{2}$, equipado com dois bicos de jato plano tipo leque XR110.03,

Tabela 1 - Características químicas do solo utilizado no experimento. Viçosa-MG, 2004

\begin{tabular}{|c|c|c|c|c|c|c|c|c|c|c|c|}
\hline $\mathrm{P}$ & K & $\mathrm{Ca}^{+2}$ & $\mathrm{Mg}^{+2}$ & $\mathrm{Al}^{+3}$ & $\mathrm{H}+\mathrm{Al}$ & $\mathrm{SB}$ & $\mathrm{t}$ & $\mathrm{T}$ & $\mathrm{V}$ & $\mathrm{m}$ & $\mathrm{pH}$ \\
\hline \multicolumn{2}{|c|}{$\left(\mathrm{mg} \mathrm{dm}^{-3}\right)$} & \multicolumn{7}{|c|}{$\left(\mathrm{cmol}_{\mathrm{c}} \mathrm{dm}^{-3}\right)$} & \multicolumn{2}{|c|}{$(\%)$} & $\left(\mathrm{H}_{2} \mathrm{O}\right)$ \\
\hline 2 & 68 & 2,25 & 0,58 & 0,0 & 3,10 & 3,00 & 3,00 & 6,10 & 49,2 & 0,0 & 6,04 \\
\hline
\end{tabular}

pH em água, $\mathrm{Kcl}$ e $\mathrm{Ca} \mathrm{CL}_{2}$ - relação 1:2,5; P e K - extrator Mehlich 1; Ca, $\mathrm{Mg}$ e Al - extrator $\mathrm{KCL}-1 \mathrm{~mol} \mathrm{l}^{-1} ; \mathrm{H}+\mathrm{Al}$ - extrator Acetato de Cálcio 0,5 mol L-1 $-\mathrm{pH} 7,0 ; \mathrm{SB}=$ soma de bases trocáveis; $\mathrm{CTC}(\mathrm{T})=$ capacidade de troca catiônica a pH 7,0; CTC $(\mathrm{t})=$ capacidade de troca catiônica efetiva; $\mathrm{V}$ = índice de saturação de bases; $\mathrm{m}$ = índice de saturação de alumínio. Análises realizadas nos laboratórios de análises físicas e químicas de solo do Dep. de Solos da UFV. 
espaçados de $0,5 \mathrm{~m}$, e pressão constante de $2,4 \mathrm{kgf} \mathrm{cm}^{-2}$, proporcionando volume de calda de $200 \mathrm{~L} \mathrm{ha}^{-1}$. À calda foi adicionado espalhante adesivo Extravon a 0,2\% (v/v). As avaliações dos sintomas de intoxicação das plantas de algodoeiro foram efetuadas aos 10, 20 e 30 DAA, atribuindo-se notas que variaram de 0 a $100 \%$, sendo 0 ausência de intoxicação e $100 \%$ morte da planta, sempre em comparação com o tratamento que não recebeu o herbicida.

Em cada época de coleta mediu-se a altura das plantas, considerando-se a distância do solo até o ápice da planta. Após o corte das plantas, ao nível do solo, fez-se a separação de suas partes: folhas, ramos, caule principal, maçãs e raízes. A área foliar foi obtida com um medidor de área foliar (Li-cor Instruments, modelo LI-3000). Todo o material colhido foi colocado em estufa com circulação forçada de ar, a $70{ }^{\circ} \mathrm{C}$ por $72 \mathrm{~h}$, sendo posteriormente pesado, exceto o algodão em caroço, que foi colhido a partir de 130 dias após o plantio. Os resultados de matéria seca e área foliar foram utilizados para se determinar a taxa de crescimento absoluto (TCA), taxa de crescimento relativo (TCR), taxa de assimilação líquida (TAL) e razão de área foliar (RAF).

A TCA (g por dia), que representa a matéria seca acumulada por intervalo de tempo, foi calculada pela fórmula TCA $=\left(\mathrm{P}_{\mathrm{n}}-\mathrm{P}_{\mathrm{n}-1}\right) /\left(\mathrm{T}_{\mathrm{n}}-\mathrm{T}_{\mathrm{n}-1}\right)$, em que $\mathrm{P}_{\mathrm{n}}$ é a matéria seca acumulada até a avaliação $\mathrm{n} ; \mathrm{P}_{\mathrm{n}-1}$ é a matéria seca acumulada até a avaliação $\mathrm{n}-1 ; \mathrm{T}_{\mathrm{n}}$ é o número de dias após o tratamento por ocasião da avaliação $n$; e $T_{n-1}$ é o número de dias após o tratamento por ocasião da avaliação n-1. A TCR expressa o crescimento da planta em um intervalo de tempo em relação à materia seca acumulada no início desse intervalo, sendo calculada pela fórmula TCR = (ln P2 - ln P1)/T2-T1 $\mathrm{g} \mathrm{g}^{-1}$ por dia. A TAL expressa a taxa de fotossíntese líquida, que é a matéria seca produzida (em gramas) por unidade de área foliar e por unidade de tempo, sendo calculada pela seguinte fórmula: TAL $=\left[\left(\mathrm{P}_{\mathrm{n}}-\mathrm{P}_{\mathrm{n}-1}\right) /\left(\mathrm{T}_{\mathrm{n}}-\mathrm{T}_{\mathrm{n}-1}\right)\right] \cdot\left[\left(1 \mathrm{n} \mathrm{A}_{\mathrm{n}}{ }^{-}\right.\right.$ $\left.\left.\ln A_{n-1}\right) /\left(A_{n}-A_{n-1}\right)\right]$, em que $A_{n}$ é a área foliar da planta por ocasião da avaliação n; e $A_{n-1}$ é a área foliar da planta por ocasião da avaliação n-1. A RAF, que representa a relação entre a área responsável pela fotossíntese e a matéria seca total produzida, foi calculada pela fórmula $\mathrm{RAF}=\mathrm{A}_{\mathrm{n}} / \mathrm{P}_{\mathrm{n}}$ (Benincasa, 2003).
Os resultados foram submetidos à análise de variância e regressão. Os modelos foram escolhidos considerando-se a significância dos coeficientes de regressão, utilizando-se o teste t a $5 \%$ de probabilidade, o coeficiente de determinação e o significado biológico.

\section{RESULTADOS E DISCUSSÃO}

A intoxicação do algodoeiro pelo trifloxysulfuron-sodium atingiu maior nivel (35\%) aos 20 DAA. Os sintomas começaram leves, aproximadamente aos 2 DAA, com manchas amarelas, e se tornaram acentuados no final da primeira semana, principalmente nas folhas jovens. Esses sintomas são típicos dos herbicidas inibidores da enzima acetolactato sintase (Porterfield et al., 2002b). Resultados de pesquisas mostram que o nível de intoxicação do algodoeiro, causado pelo trifloxysulfuron-sodium, é variável (Burke et al., 2002; Schraer et al., 2002; Porterfield et al., 2002b; Freitas et al., 2004), principalmente em função da dose e do estádio de desenvolvimento da cultura.

A altura das plantas foi avaliada até os 52 DAA, sendo, nesse período, sempre menor para as plantas submetidas ao herbicida (Figura 1A). Todavia, estudos realizados por Miller et al. (2002) mostraram que o trifloxysulfuron-sodium não afetou a altura final do algodoeiro e que diversos fatores, como o cultivar e as condições ambientais, podem ter contribuído para redução na altura das plantas.

O acúmulo de matéria seca do caule principal, do total de folhas, da folha do caule principal, da parte aérea e a área foliar aumentaram com o desenvolvimento das plantas (Figura 1B, C, D, E e F). Entretanto, o ponto de máximo crescimento para essas características foi atingido mais precocemente nas plantas que não foram submetidas ao herbicida (plantas-controle), exceto para área foliar, que atingiu o máximo de $3.025 \mathrm{~cm}^{2}$ por planta aos 63 DAA nas plantas-controle, enquanto nas plantas submetidas ao herbicida ela foi de $3.306,0 \mathrm{~cm}^{2}$ por planta aos 57 DAA (Figura 1E). Um dos fatores que contribuíram para esse comportamento foi o maior número de folhas nos ramos, observado nas plantas que receberam o herbicida. Esses resultados 

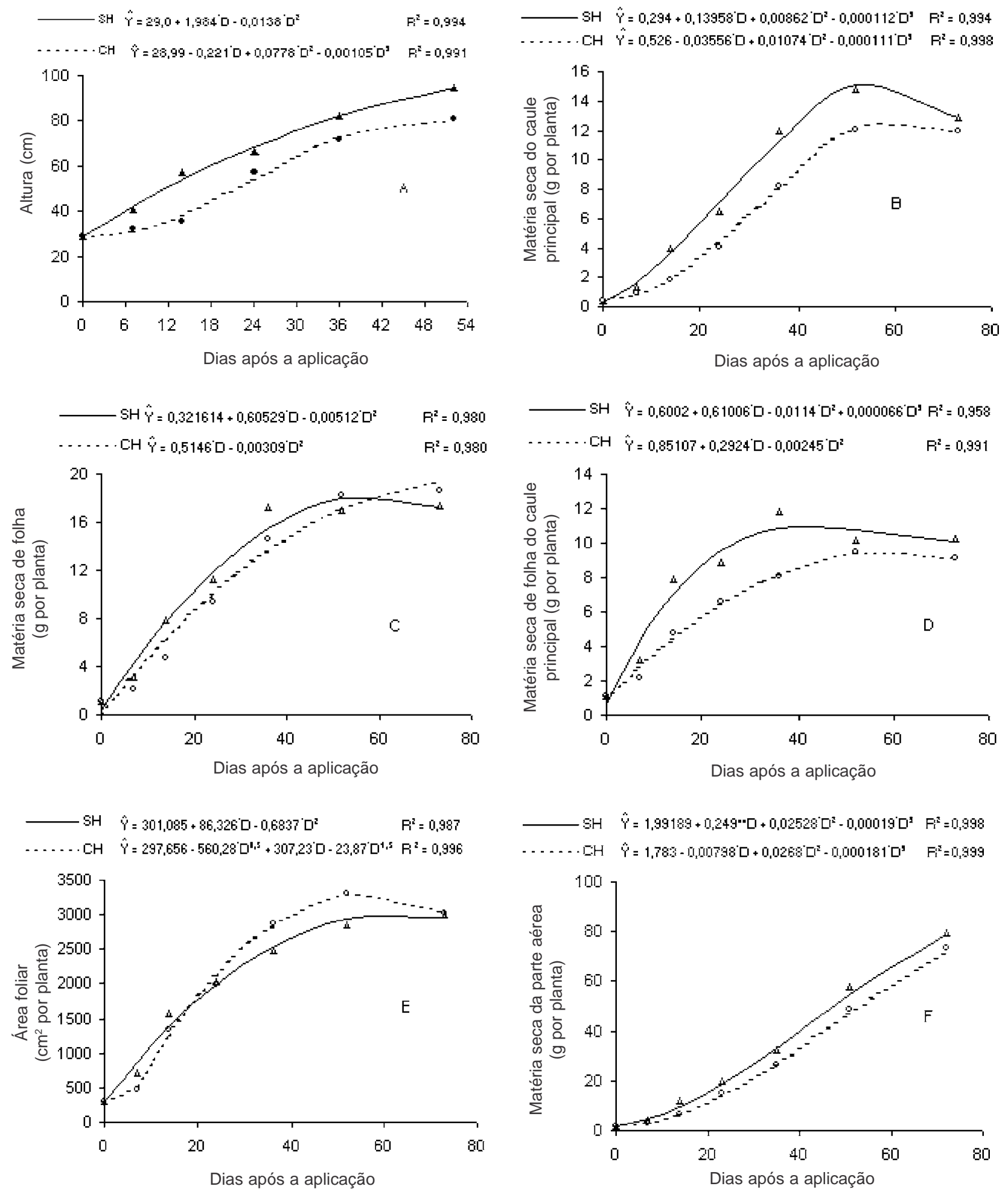

Figura 1 - Altura (A), matéria seca de caule principal (B), matéria seca de folha (C), matéria seca de folha do caule principal (D), área foliar (E) e matéria seca da parte aérea $(\mathrm{F})$ do algodoeiro submetido $(\mathrm{CH})$ ou não $(\mathrm{SH})$ a 7,5 $\mathrm{g} \mathrm{ha}^{-1}$ de trifloxysulfuronsodium, em função de dias após a aplicação.

Planta Daninha, Viçosa-MG, v. 24, n. 1, p. 123-129, 2006 
corroboram os de outro experimento, realizado em condições similares, em que se obteve aumento da área foliar e do número de folhas com o aumento da dose de trifloxysulfuronsodium até $11,25 \mathrm{~g} \mathrm{ha}^{-1}$, aos $35 \mathrm{DAA}$. O acúmulo de matéria seca pelo caule principal foi menor nas plantas que receberam trifloxysulfuron-sodium, com produção máxima observada aos 63 DAA (Figura 1B). Nas plantas-controle, a produção máxima de matéria seca foi verificada aos 58 DAA (Figura 1B). A pequena redução de produção de matéria seca observada a partir desses períodos pode estar ligada a alguma variação na uniformidade das plantas entre as avaliações. A maior produção de matéria seca de caule das plantas-controle até a última avaliação ocorreu, principalmente, pelo maior crescimento em altura e no diâmetro destas na fase inicial de desenvolvimento e, posteriormente, apenas pela maior altura das plantas.

A produção de matéria seca de folha das plantas-controle foi máxima aos 59 DAA e a das plantas submetidas ao herbicida aumentou até a última avaliação, realizada aos 73 DAA (Figura 1C). Aos 59 DAA foi verificada produção semelhante de matéria seca pelas plantas-controle e pelas plantas submetidas ao trifloxysulfuron-sodium. No entanto, quando se analisou a produção de matéria seca de folha do ramo principal, verificou-se menor produção de matéria seca nas plantas submetidas ao herbicida até a última avaliação (Figura 1D). O ponto de máxima produção de matéria seca das folhas do caule principal nas plantas-controle foi aos 36 dias após aplicação dos tratamentos, e nas plantas tratadas com 7,5 $\mathrm{g} \mathrm{ha}^{-1}$ de trifloxysulfuron-sodim, aos 59 DAA. As plantas que receberam o herbicida apresentaram menor acúmulo de matéria seca da parte aérea até os 73 DAA (Figura 1 F), sem que reduções fossem verificadas na produção de algodão em caroço, embora houvesse tendência de menor produção (Tabela 2).

A velocidade de acúmulo de matéria seca atingiu o ponto de máximo aos 44 dias da aplicação dos tratamentos nas plantas-controle e aos 52 DAA nas plantas que foram submetidas a 7,5 $\mathrm{g} \mathrm{ha}^{-1}$ de trifloxysulfuron-sodium (Figura 2A). A redução na taxa de crescimento a partir desses períodos coincidiu com o período de florescimento e formação das maçãs, que são drenos efetivos da planta. Desse modo, a redução na taxa de crescimento pode estar relacionada ao menor investimento da planta na produção de folhas e ao envelhecimento destas, que, segundo Muramoto et al. (1967), limita sua atividade fotossintética. Na Tabela 2 , verifica-se menor acúmulo de matéria seca de maçãs aos 52 e 72 DAA para as plantas submetidas a 7,5 $\mathrm{g} \mathrm{ha}^{-1}$ de trifloxysulfuronsodium. Aos 52 DAA, o acúmulo de biomassa das maçãs das plantas-controle foi, aproximadamente, o dobro em relação àquelas submetidas ao herbicida. Contudo, aos 73 DAA, essa diferença foi bem menor (21\%), evidenciando a recuperação das plantas. O atraso na formação das maçãs - redução na sua massa sob efeito do herbicida - ocorre, possivelmente, devido aos efeitos do herbicida sobre o algodoeiro e ao tempo necessário para recuperação das plantas. O menor crescimento das folhas, principalmente aquelas do ramo principal, que são responsáveis pela maior parte da produção de fotoassimilados destinados aos dois primeiros frutos de cada ramo frutífero (Beltrão \& Azevedo, 1993), pode ter contribuído para o menor desenvolvimento inicial das maçãs.

As plantas-controle apresentaram TCR superior à das plantas submetidas ao herbicida trifloxysulfuron-sodium nas primeiras avaliações, realizadas aos 7 e aos 14 DAA, porém em ambos os tratamentos houve redução desta taxa ao longo do ciclo do algodoeiro (Figura 2B). Tal redução contínua da TCR pode ser explicada pelo crescimento da planta, que implica maior gasto com a manutenção das estruturas, resultando em maior respiração de manutenção (Pereira, 1989). Como é necessário maior alocação de fotoassimilados para

Tabela 2 - Matéria seca de maçãs aos 52 e 72 dias após a aplicação do herbicida e produção de algodão em caroço (g por planta) em algodoeiro submetido ou não ao trifloxysulfuron-sodium. Viçosa-MG, 2004

\begin{tabular}{|c|c|c|c|}
\hline \multirow{2}{*}{$\begin{array}{c}\text { Trifloxysulfuron- } \\
\text { sodium }\left(\mathrm{g} \mathrm{ha}^{-1}\right)\end{array}$} & 52 DAA & 72 DAA & Colheita \\
\cline { 2 - 4 } & Maçãs & Maçãs & $\begin{array}{c}\text { Algodão em } \\
\text { caroço }\end{array}$ \\
\hline 0,0 & $19,75 \mathrm{a}$ & $47,29 \mathrm{a}$ & $53,89 \mathrm{a}$ \\
\hline 7,5 & $9,13 \mathrm{~b}$ & $37,17 \mathrm{~b}$ & $48,75 \mathrm{a}$ \\
\hline
\end{tabular}

Médias seguidas pela mesma letra, nas colunas, não diferem entre si a $5 \%$ de probabilidade pelo teste $\mathrm{F}$. 
essa função, sua disponibilidade é reduzida para o crescimento. Outro fator que contribuiu para a redução da TCR foi o auto-sombreamento, cuja importância aumentou com a idade da planta. Além disso, na fase final da cultura, há redução na taxa de crescimento, em razão da morte de folhas e gemas (Milthorpe \& Moorby, 1974, citados por Urchei et al., 2000).

Semelhantemente à TCR, a TAL foi superior nas avaliações iniciais para as plantas-controle e apresentou maior taxa de redução ao longo do ciclo fenológico da cultura (Figura 2C). Esses resultados representam o balanço entre a matéria produzida pela fotossíntese e a consumida através da respiração e, portanto, a eficiência das folhas na produção de matéria seca, possibilitando estimar a fotossíntese líquida (Pereira \& Machado, 1987). As maiores TCR e TAL das plantas-controle nas primeiras avaliações indicam o efeito do herbicida na redução do crescimento do algodoeiro nos primeiros 15 dias após sua aplicação.

A razão de área foliar (RAF), que é a área foliar útil para a fotossintese, reduziu com o desenvolvimento da cultura (Figura 2D), em decorrência do surgimento de tecidos e estruturas que são drenos altamente competitivos, como flores, maçãs e sementes, além do autosombreamento (Urchei et al., 2000; Benincasa, 2003), indicando que, progressivamente, a
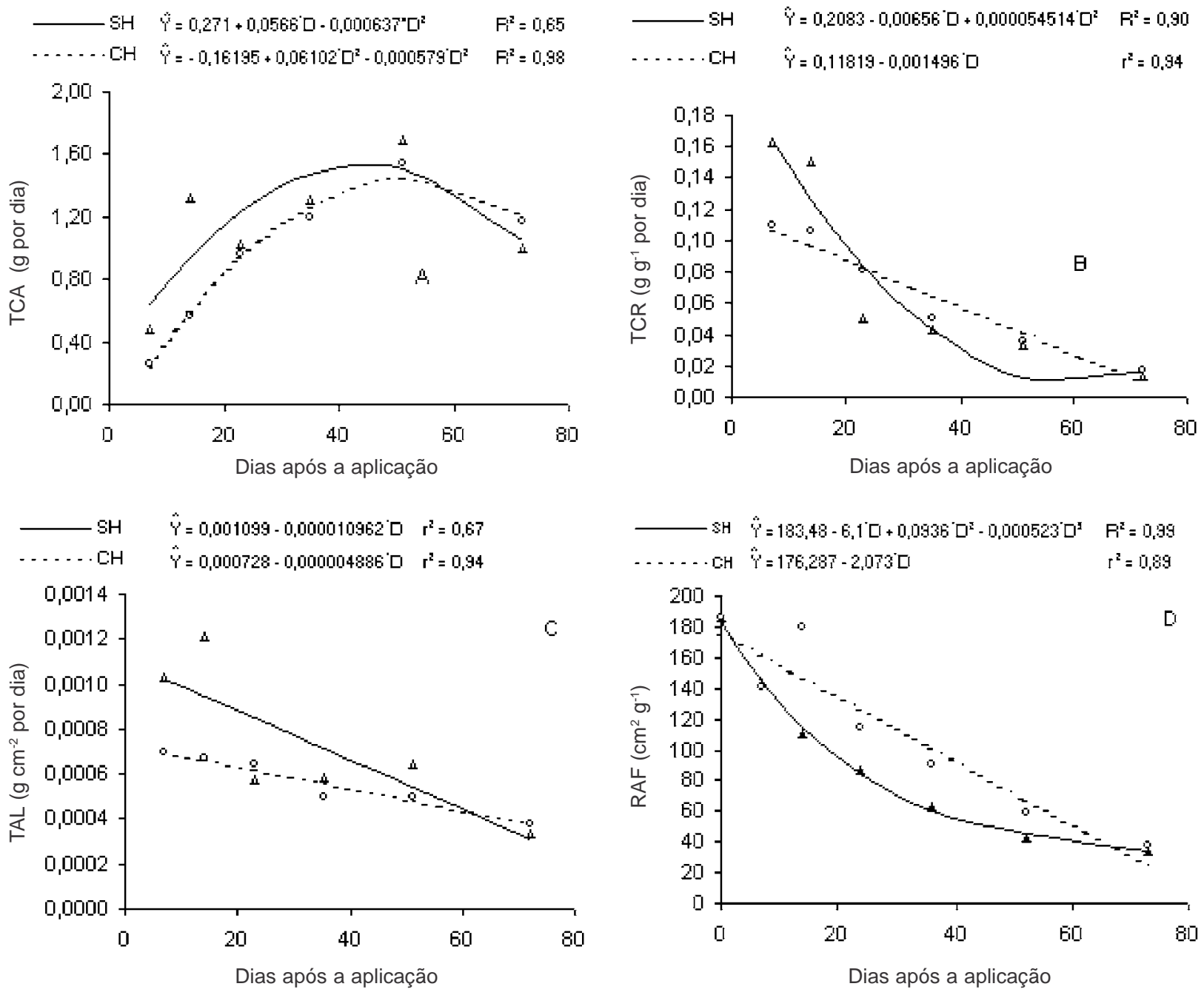

Figura 2 - Taxa de crescimento absoluto (A), taxa de crescimento relativo (B), taxa assimilatória líquida (C) e razão de área foliar (D) do algodoeiro submetido $(\mathrm{CH})$ ou não $(\mathrm{SH})$ a 7,5 $\mathrm{g} \mathrm{ha}^{-1}$ de trifloxysulfuron-sodium, em função de dias após sua aplicação. 
quantidade de fotoassimilados destinada às folhas diminuiu. Observa-se que a RAF declina já nas primeiras avaliações, sendo isso perfeitamente compreensivel, pois as avaliações começaram aos 19 dias após a emergência, quando possivelmente as folhas superiores já estavam causando interferência (auto-sombreamento) sobre as folhas inferiores; segundo Benincasa (2003), a tendência é a área útil diminuir, a partir de uma certa fase. Ademais, a planta de algodão apresenta estrutura planofilar, o que intensifica o efeito de autosombreamento. As plantas-controle apresentaram redução mais acentuada na RAF que as submetidas ao herbicida, sendo similar na última avaliação (Figura 2D). Isso ocorreu, provavelmente, em razão da maior eficiência das folhas das plantas-controle em converter energia luminosa e $\mathrm{CO}_{2}$ em matéria seca, já que a RAF é a área foliar usada pela planta para produzir um grama de matéria seca (Benincasa, 2003); isso é evidenciado, principalmente, pelo maior acúmulo de matéria seca da parte aérea pelas plantas-controle (Figura $1 \mathrm{~F}$ ).

O trifloxysulfuron-sodium afetou o desenvolvimento do algodoeiro, principalmente nas primeiras semanas após sua aplicação. No entanto, a rápida recuperação da planta tratada possibilitou produção de algodão em caroço semelhante àquela obtida com as plantas-controle.

\section{LITERATURA CITADA}

BARBER, L. T. et al. Weed control with GGA-362622 in Roundup Ready and BXN Cotton systems. In: SOUTHERN WEED SCIENCE SOCIETY, 55., 2002, Atlanta.

Proceedings... Atlanta: Hyatt Regency, 2002. p. 140-141.

BELTRÃO, N. E. M.; AZEVEDO, D. M. P. Defasagem entre as produtividades real e potencial do algodoeiro herbáceo: limitações morfológicas, fisiológicas e ambientais. Campina Grande: EMBRAPA-CNPA, 1993. 108 p. (EMBRAPA-CNPA. Documentos, 39).

BENINCASA, M. P. Análise de crescimento de plantas (noções básicas). Jaboticabal: Funep, 2003. 41 p.

BURKE, I. C. et al. Weed management in cotton with CGA 362622 e pyrithiobac systems. In: SOUTHERN WEED SCIENCE SOCIETY, 55., 2002, Atlanta. Proceedings... Atlanta: Hyatt Regency, 2002. p. 28-29.
FREITAS, R. S. et al. Tolerância do algodoeiro a trifloxysulfuron sodium. In: CONGRESSO BRASILEIRO DA CIÊNCIA DAS PLANTAS DANINHAS, 24., 2004, São Pedro-SP. Anais... São Pedro: SBCPD, 2004. p. 175. CD-ROM.

KVET, J. et al. Methods of growth analysis. In: SESTÁK, Z.; CATSKÝ, J.; JARVIS, P. G. Plant photosynthetic production: manual of methods. The Hague: W. Junk, 1971. p. 343-391.

MARUR, C. J.; RUANO, O. Escala do algodão: um sistema de referência para determinação de fases de crescimento e desenvolvimento do algodoeiro. R. Cultivar, v. 4, p. 16-17, 2002.

MILLER, D. K. et al. Weed control and cotton tolerance with CGA 362622. In: SOUTHERN WEED SCIENCE SOCIETY, 55., 2002, Atlanta. Proceedings... Atlanta: Hyatt Regency, 2002. p. 30.

MURAMOTO, H.; HESKETH, J. D.; ELMORE, C. D. Leaf growth, leaf aging and leaf photosynthetic rates of cotton plants. In: BELTWIDE COTTON PRODUCTION RESEARCH CONFERENCES, 1967, Dallas, Texas. Proceedings... Memphis: National Cotton Council, 1967. p. 161-165.

PEREIRA, A. R.; MACHADO, E. C. Análise quantitativa do crescimento de comunidades vegetais. Campinas: IAC, 1987. 33 p. (IAC. Boletim Técnico, 114).

PEREIRA, A. R. Aspectos fisiológicos da produtividade vegetal. R. Bras. Fisiol. Veg., v. 1, n. 2, p. 139-142, 1989.

PORTERFIELD, D.; WILCUT J. W.; ASKEW, S. D. Weed management with CGA-362622, fluometuron, and prometryn in cotton. Weed Sci., v. 50, n. 5, p. 642-647, 2002 a.

PORTERFIELD, D. et al. Weed free response of seven cotton (Gossypium hirsutum) cultivars to CGA-362622 postemergence. Weed Technol., v. 16, n. 1, p. 180-183, $2002 b$.

SILVA, N. M. Nutrição mineral e adubação do algodoeiro. In: CIA, E.; FREIRE, E. C.; SANTOS, W. J. (Eds.). Cultura do algodoeiro. Piracicaba: POTAFOS, 1999. p. 57-92.

SCHRAER, S. M. et al. Cotton response to GGA-362622: rates, timing, and tank-mixtures. In: SOUTHERN WEED SCIENCE SOCIETY, 55., 2002, Atlanta. Proceedings... Atlanta: Hyatt Regency, 2002. p. 139-140.

URCHEI, M. A.; RODRIGUES, J. D.; STONE, L. F. Análise de crescimento de duas cultivares de feijoeiro sob irrigação, em plantio direto e preparo convencional. Pesq. Agropec. Bras., v. 35, n. 3, p. 497-506, 2000. 